\title{
Dermatan sulfate remodeling associated with advanced Dupuytren's contracture
}

\author{
Ewa M. Koźma ${ }^{1 \bowtie}$, Andrzej Głowacki ${ }^{1}$, Krystyna Olczyk ${ }^{1}$ and Magdalena Ciecierska ${ }^{2}$ \\ ${ }^{1}$ Department of Clinical Chemistry and Laboratory Diagnostics, Medical University of Silesia, Sosnowiec, \\ Poland; ${ }^{2}$ Division of Orthopaedic Surgery, First Municipal Hospital, Sosnowiec, Poland
}

Received: 11 July, 2007; revised: 27 September, 2007; accepted: 12 November, 2007 available on-line: 09 December, 2007

\begin{abstract}
Dermatan sulfate (DS) widespread as a component of extracellular matrix proteoglycans, is characterized by great bio-reactivity and remarkable structural heterogeneity due to distinct degrees of sulfation and glucuronosyl epimerization and different polymerization degrees. However, DS metabolism under various biological conditions is poorly known. Dupuytren's contracture is a benign fibromatosis leading to complex remodeling of the palmar fascia structure and properties. However, it remains unclear whether the disease affects the structure of DS, which is the major tissue glycosaminoglycan. Thus the aim of the study was to examine the structure of the total DS in Dupuytren's fascia. DS chains were extracted from 5 samples of normal fascia and 7 specimens of Dupuytren's tissue by papain digestion followed by fractionation with cetylpyridinium chloride. Then, DS structure analysis was performed comprising the evaluation of its molecular masses and sensitivity to hyaluronidase and chondroitinase B. Dupuytren's contracture is associated with significant remodeling of DS chain structure revealed by (1) a distinct profile of chain molecular masses characterized by the appearance of long size components as well as the increase in the content of small size chains; (2) a different glucuronosyl epimerization pattern connected with the enhanced content of glucuronate disaccharide blocks; (3) chain oversulfation. These structural alterations in total DS may modify the GAG interactions especially affecting collagen fibrillogenesis and growth factor availability. Thus, Dupuytren's contracture associated DS remodeling may promote the phenomena typical for advanced disease: apoptosis and reduction in cell number as well as the appearance of dense pseudotendinous collagen matrix.
\end{abstract}

Keywords: dermatan sulfate, epimerization, fibrous cords, Dupuytren's contracture

\section{INTRODUCTION}

Dermatan sulfate (DS) is a glycosaminoglycan (GAG) widespread in animal tissues as a component of extracellular matrix (ECM) glycoproteins termed dermatan sulfate proteoglycans (DSPGs). Their linear DS chains covalently linked to various core proteins are polymers of disaccharide units consisting of $N$-acetyl-galactosamine and hexuronic acid (D-glucuronate or L-iduronate) residues, most frequently existing as sulfated derivatives (Prydz \& Dalen, 2000). High content of negative charge enables DS to interact with miscellaneous bioligands such as ECM molecules (fibronectin and other fibrillar and nonfibrillar proteins), proteases and their effectors as well as cytokines, growth factors and their receptors (Wegrowski \& Maquart, 2004). However, DS chain binding affinity to these molecules can change under different physiological and pathological conditions due to the GAG structural heterogeneity connected

\footnotetext{
${ }^{\square}$ Corresponding author: Ewa M. Koźma, Department of Clinical Chemistry and Laboratory Diagnostics, Medical University of Silesia, Jagiellońska 4, 41-200 Sosnowiec, Poland; tel./fax: (48) 32364 1150; e-mail: mkozma@slam.katowice.pl Abbreviations: CPC, cetylpyridinum chloride; CS, chondroitin sulfate; DC, Dupuytren's contracture; DF, Dupuytren's fascia; DS, dermatan sulfate; DSPG, dermatan sulfate proteoglycan; ECM, extracellular matrix; FGF-2, fibroblast growth factor-2; GAG, glycosaminoglycan; GlcA, glucuronate; HA, hyaluronan; IdoA, iduronate; NF, normal fascia; PAGE, polyacrylamide gel electrophoresis; PDGF, platelet-derived growth factor; PG, proteoglycan; TGF $\beta$, transforming growth factor $\beta$.
} 
with differential degrees of monosaccharide residue sulfation and glucuronosyl epimerization (conversion of glucuronate residue to iduronate residue) (Prydz \& Dalen, 2000). The latter modification determines DS chain flexibility thus influencing the GAG bio-reactivity (Casu et al., 1993). Both features of DS, i.e. the remarkable structural heterogeneity of its chains as well as its profound ability to bind other molecules promote DSPGs as regulating/controlling factors involved in various biological processes such as wound healing or organ/tissue fibrosis. An example of the latter disease is Dupuytren's contracture (DC). The disease classified as a benign fibromatosis of palmar fascia (Rayan, 1999) affecting mainly men particularly the elderly ones (Gudmundsson et al., 2000), displays differentiated prevalence. In Central Europe the disease frequency is estimated at 6.6\% (Jung et al., 1971). In contrast, the highest prevalence of DC is reported in Scandinavia and Scotland where in some populations almost $40 \%$ of elderly men have some symptoms of the disease (Lennox et al., 1993; Gudmundsson et al., 2000). Nevertheless, a more severe form of the disease, characterized by significant finger contracture, is found in $5.0 \%$ of Scandinavian men and only $1.4 \%$ of them require operative treatment (Gudmundsson et al., 2000).

In its early stages, DC is distinguished by the presence of nodules containing (myo)fibroblasts which demonstrate intensive proliferation activity (Rayan, 1999; Tomasek et al., 1999). Moreover, some of these cells exhibit distinct chromosomal abnormalities (trisomies 7 and 8) (Dal Cin et al., 1999). However, the disease progress is connected with remarkable reduction in cellularity and the appearance of dense pseudotendinous collagenous cords leading to contracture of the digits (Rayan, 1999). Both above mentioned lesions usually co-exist in affected palmar fascia (Rayan, 1999). In DC development, growth factors such as transforming growth factor $\beta$ (TGF $\beta$ ) and platelet-derived growth factor (PDGF) play a key role (Badalamente \& Hurst, 1999). On the other hand, as it has been revealed in in vitro studies, these molecules influence glycosaminoglycan metabolism also affecting DS structure (Kinsella et al., 2004; Tiedemann et al., 2005). Indeed, an accumulation of DS and chondroitin sulfate (CS) is observed in Dupuytren's fascia (Flint et al., 1982; Tunn et al., 1988). However, the detailed aspects of disease-associated metabolism of DS as the major GAG both of normal and Dupuytren's fascia ECM (Flint et al., 1982) is poorly known. Our previous study concerned the structure of DS chains derived from particular PGs extractable with urea from Dupuytren's fascia fibrous cords (Koźma et al., 2005). However, this DS fraction represents only a minor part of total DS of fibrous cords (Koźma et al., 2005). Thus it prompts us to evaluate the structure of the latter. To our knowledge the epimerization pattern of total DS of Dupuytren's fascia fibrous cords is analyzed here for the first time.

\section{MATERIALS AND METHODS}

Materials. Glycine, papain, acrylamide, Azur A, dimethyl sulfoxide, chondroitinase ABC [EC 4.2.2.4] from Proteus vulgaris, chondroitinases AC I [EC 4.2.2.5] and B as well as heparinase III (heparitinase I) [EC 4.2.2.8] from Flavobacterium heparinum, bovine testicular hyaluronidase [EC 3.2.1.35] were supplied by Sigma-Aldrich Chemie GmbH (Steinheim, Germany). Cellogel cellulose acetate, Tris, $N, N^{\prime}$-methylenebisacrylamide as well as reference glycosaminoglycans (DS from pig skin, C-4-S from whale cartilage, C-6-S from shark cartilage, HA from rooster comb) were obtained from Serva Feinbiochemica GmbH (Heidelberg, Germany). Cetylpyridinium chloride was provided by Loba-Chemie (Wien-Fischamend, Austria). Alcian blue 8GS was purchased from Aldrich Chemie (Steinheim, Germany). $N, N, N^{\prime}, N^{\prime}$-tetramethyl-ethylenediamine was supplied by ICN Biomedicals, Inc. (Aurora, OH, USA). All other chemicals were supplied by POCh (Gliwice, Poland) and were of analytical grade.

Tissue material. Specimens of normal palmar fascia (NF) were collected from 5 healthy men (age range 44 to 50) during reconstruction surgery following accidental injury. The samples of primary Dupuytren's fascia (DF) were obtained from 7 patients of matched sex and age, treated operatively for the disease. From each DF sample, the regions corresponding to fibrous lesions according to criteria set by Rombouts et al. (1989) were collected. All tissue specimens were obtained with informed consent of the patients. The study protocol was approved by the Regional Ethics Committee. The samples, freed from adjacent tissues, were stored at $-20^{\circ} \mathrm{C}$ until processing.

Isolation and fractionation of DS. The isolation and fractionation of NF and DF DSs were carried out as described previously (Głowacki et al., 1994; Koźma et al., 2000). Briefly, tissue samples after homogenization with acetone and weighing were digested with papain to release GAG chains from PG core proteins. Both peptides generated by papain action and proteins resistant to the enzyme were removed by precipitation with trichloroacetic acid. Subsequently, GAGs were dialyzed, precipitated with ethanol, dissolved in $0.5 \mathrm{M}$ potassium acetate and reprecipitated. Obtained GAGs containing mainly DS chains were further fractionated with cetylpyridinium chloride (CPC). As the result of the applied procedure three DS fractions were obtained: fraction I comprised this GAG chains forming CPC-complex- 
es soluble in $0.02 \mathrm{M} \mathrm{Na}_{2} \mathrm{SO}_{4}$ and $1.5 \%(\mathrm{w} / \mathrm{v}) \mathrm{CPC}$ solution, fraction II contained DS chains precipitating in $0.07 \%(\mathrm{w} / \mathrm{v}) \mathrm{CPC}$ and $0.2 \mathrm{M} \mathrm{MgCl}_{2}$ solution, whereas fraction III included DS chains precipitating in $0.09 \%(\mathrm{w} / \mathrm{v}) \mathrm{CPC}$ and $0.06 \mathrm{M} \mathrm{MgCl}_{2}$ solution. Hexuronic acid content was measured in these fractions (Blumenkrantz \& Asboe-Hansen, 1973). Moreover, to evaluate the purity of these DS fractions their samples were analyzed by cellulose acetate electrophoresis before and after specific degradation as described previously (Koźma et al., 2000).

Analysis of DS structure. The glucuronosyl epimerization patterns of DSs derived from NF and DF were evaluated on the basis of the sensitivity of these GAGs to testicular hyaluronidase as well as chondroitinase B action with subsequent resolution of enzyme resistant chain fragments by polyacrylamide-gradient-gel electrophoresis (gradient PAGE) as described earlier (Koźma et al., 2001). Briefly, after the elimination of heparan sulfate contamination by sequential action of heparinase III and nitrous acid, GAGs of fractions I and II were submitted to alkaline treatment to remove core protein fragments. Subsequently, GAG samples were treated with hyaluronidase or chondroitinase B. The degradation of DS by the former enzyme was performed in $0.1 \mathrm{M}$ sodium acetate buffer, $\mathrm{pH}$ 5.0, containing $0.15 \mathrm{M} \mathrm{NaCl}$ for $24 \mathrm{~h}$, at $37^{\circ} \mathrm{C}$ (Cowman et al., 1984) while GAG cleavage by chondroitinase $\mathrm{B}$ was performed in $0.05 \mathrm{M}$ Tris/ $\mathrm{HCl}$ buffer, $\mathrm{pH} 7.5$, for $24 \mathrm{~h}$, at $25^{\circ} \mathrm{C}$ as recommended by the suppliers. However, samples of fraction II due to small though significant chondroitin sulfate (CS) content were subjected to the procedure of selective DS precipitation with $27.5 \%$ ethanol in $5 \%$ calcium acetate and $0.5 \mathrm{M}$ acetic acid (Roden et al., 1972) before chondroitinase B treatment. Further identification of chondroitinase B resistant DS chain fragments was performed by the evaluation of their sensitivity to chondroitinase AC I (Koźma et al., 2001). All obtained degradation products were then analyzed electrophoretically.

To estimate their molecular masses, intact DS chains of fractions I and II were subjected to polyacrylamide-gradient-gel electrophoresis simultaneously with standard DS. Moreover, fraction I samples treated with chondroitinase $\mathrm{ABC}$ in $0.05 \mathrm{M}$ Tris/ $\mathrm{HCl}$ buffer, $\mathrm{pH} 8.0$ for $2 \mathrm{~h}$, at $37^{\circ} \mathrm{C}$ (Hamai et al., 1997) were electrophoresed. This step allowed to show the interference of non-DS/CS glycans in the intact DS chain electrophoretic profile.

Polyacrylamide-gradient-gel electrophoresis of DS and products of its degradation. Intact DS chains and products of their enzymatic depolymerization were separated on the basis of their molecular size by gradient-PAGE as previously described (Koźma et al., 2001). After the resolution, the gels were fixed and stained, first with Azure A and then with ammoniacal silver nitrate (Lyon \& Gallagher, 1990), and subjected to densitometry. The employed procedure uses a small amount of glycan material and was chosen due to the limited amount of control tissue. However, the used staining method makes it possible to visualize only oligosaccharides larger than tetrasaccharides due to their sufficiently great electric charge (Lyon \& Gallagher, 1990).

Statistical analysis. The data were analyzed with the Shapiro-Wilk test to verify the assumption of normal distribution. The results were expressed as mean values \pm S.D. Statistical evaluation of GAG content was performed with the Student's $t$ test, accepting $P<0.05$ as significant. Between-group comparisons of chondroitinase $B$ resistant region contribution to DS chain structure were based on one-way ANOVA and Duncan test.

\section{RESULTS}

\section{Characterization of DS fractions I-III and total DS content in normal and Dupuytren's fascia}

The electrophoretic analysis (not shown) disclosed that irrespectively of fascia type, each of the three fractions of glycans separated on the basis of their solubility at different ionic strength and CPC concentration, contained DS as the main component and small amounts of other molecules of saccharide origin (Table 1). Moreover, DS distribution patterns between these fractions were similar for both fascia types - most of the GAG chains were found in fraction II, while fraction III comprised the smallest amount of DS (Fig. 1). Nevertheless, each of the three glycan fractions derived from DF contained significantly more DSs than appropriate fractions from NF and mutual proportions between fraction content were also altered. Moreover, total DS content in DF, estimated as a sum of the GAG content in fractions I-III, showed a twofold increase as compared to NF (Fig. 1).

\section{Evaluation of DS structure}

The presence of DS chains in all glycan fractions I-III suggests remarkable structural heterogeneity of this GAG derived from both NF and DF. To verify this hypothesis both tissue DSs were subjected to structural analysis including evaluation of chain molecular mass as well as the pattern of glucuronosyl epimerization. However, only DSs of fractions I and II were examined due to their sufficient amounts.

The characterization of DS chain molecular weight was performed by means of gradient-PAGE. 
Table 1. Relative contribution of various glycosaminoglycan subclasses to the composition of dermatan sulfate (DS) fraction I - III derived from normal (NF) and Dupuytren's fascia (DF).

DS chains released from NF and DF samples after papain action were separated with cetylpyridinium chloride into three fractions. The purity of these fractions was evaluated by electrophoresis on cellulose acetate before and after action of chondroitinases ABC, AC I and B as well as heparinase III and nitrous acid, followed by densitometric analysis. The content of particular glycosaminoglycans is presented as the percentage of the total hexuronic acid content in individual fractions.

\begin{tabular}{lcccccc}
\hline Type of glycan & \multicolumn{2}{c}{ Fraction I } & \multicolumn{2}{c}{ Fraction II } & \multicolumn{2}{c}{ Fraction III } \\
\cline { 2 - 7 } & NF & DF & NF & DF & NF & DF \\
\hline Dermatan sulfate & $62.5 \% \pm 11.8$ & $73.5 \% \pm 5.5$ & $96.8 \% \pm 1.4$ & $86.9 \% \pm 2.5$ & $61.6 \% \pm 23.6$ & $72.4 \% \pm 11.5$ \\
Heparan sulfate & $37.1 \% \pm 11.7$ & $25.5 \% \pm 5.9$ & - & - & - & - \\
Chondroitin sulfate & - & - & $3.2 \% \pm 1.3$ & $13.1 \% \pm 2.5$ & - & - \\
$\begin{array}{l}\text { Hyaluronan } \\
\text { Keratan sulfate and non-glycosamino- } \\
\text { glycan components }\end{array}$ & $0.4 \% \pm 0.09$ & $1.0 \% \pm 0.17$ & - & - & $38.4 \% \pm 23.6$ & $27.6 \% \pm 11.5$ \\
\hline
\end{tabular}

ND, not determined; "-“ not present

The typical electrophoretic profiles of DSs from NF and DF are shown in Fig. 2a, lanes 1, 3 and in Fig. $2 \mathrm{~b}$, lanes 1,2 . Regardless of the tissue and glycan fraction, DS chains migrated in the gel as broad polydispersive bands. However, slightly higher heterogeneity of chain molecular mass was observed for DS of fraction I (Fig. 2a, lanes 1, 3). Its chains have molecular mass ranging from 5 to $22 \mathrm{kDa}$ as compared to simultaneously electrophoresed reference DS (Fig. 2a, lane 5). However, despite similarity in apparent molecular mass ranges of fraction I DSs, in NF samples the high-molecular-mass chains prevailed while in DF samples the contribution of low and high-molecular-mass species was almost equal (Fig. 2a, lanes 1, 3).

Fraction II DS chains display molecular mass ranging from 10 to $22 \mathrm{kDa}$ in the case of $\mathrm{NF}$ sam-

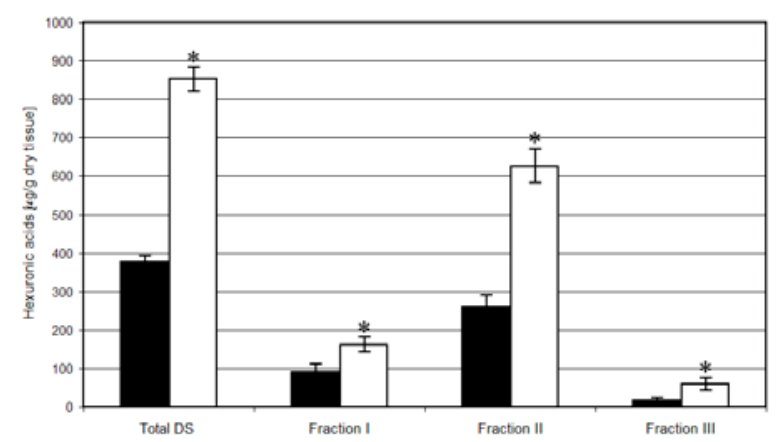

Figure 1. Total dermatan sulfate (DS) content in normal (NF) and Dupuytren's fascia (DF) as well as the distribution of these molecules between the three fractions obtained after DS fractionation with cetylpyridinium chloride.

Error bars show the mean \pm S.D. of analysis of five and seven samples of NF and DF, respectively. Black bars, DS from NF; white bars, DS from DF.* $P<0.001$.

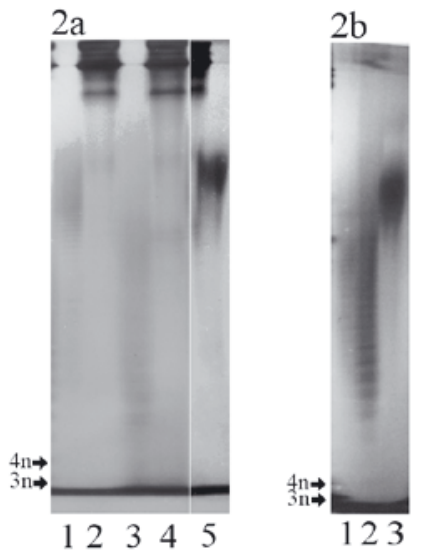

Figure 2 a-b. Size evaluation of dermatan sulfate (DS) chains from normal fascia (NF) and Dupuytren's fascia (DF).

Fraction I and fraction II DS chains were submitted to gradient-gel-electrophoresis followed by Azure A/ammoniacal silver staining. However, to evaluate the interference of other glycan contaminations on the electrophoretic profile of fraction I DSs, intact samples of this fraction were electrophoresed in parallel with the samples treated with chondroitinase $\mathrm{ABC}$. The involvement of chondroitin sulfate (CS) contamination in the electrophoretic pattern of fraction II DSs was impossible to estimate. Nevertheless, it can be assumed that CS interference is very small if not negligible, taking into account little CS content in relation to the DS one, as well as the small amount of glycan material subjected to electrophoresis. (2a) Electrophoresis of intact GAG chains from fraction I: lane 1, DS and chondroitinase $A B C$ resistant material from NF; lane 2, chondroitinase $A B C$ resistant material from NF; lane 3, DS and chondroitinase $\mathrm{ABC}$ resistant material from DF; lane 4, chondroitinase $\mathrm{ABC}$ resistant material from $\mathrm{DF}$, lane 5, standard DS with molecular masses ranging from 11 to 25 $\mathrm{kDa}$. (2b) Electrophoresis of intact DS chains from fraction II: lane 1, DS from NF; lane 2, DS from DF; lane 3, standard DS with molecular masses ranging from 11 to $25 \mathrm{kDa}$. The arrows indicate migration positions of saccharides (most probably hexa- (3n) and octasaccharides (4n)) obtained by the complete degradation of standard chondroitin-4-sulfate by testicular hyaluronidase. 


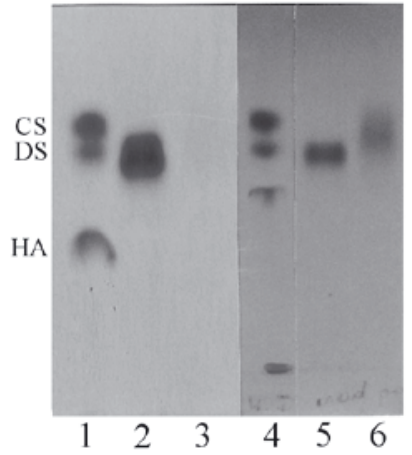

Figure 3. Separation of fraction II dermatan sulfate (DS) from chondroitin sulfate contamination.

Fraction II samples were subjected to the procedure of selective DS precipitation with $27.5 \%$ or $50 \%$ ethanol in $5 \%$ calcium acetate and $0.5 \mathrm{M}$ acetic acid followed by electrophoresis on cellulose acetate in $0.034 \mathrm{M} \mathrm{Al}_{2}\left(\mathrm{SO}_{4}\right)_{3}$ of obtained pellets (precipitates). Gels were stained with Alcian blue. Lanes 1 and 4, standard glycosaminoglycans (GAGs): CS, chondroitin sulfate, DS, dermatan sulfate, HA, hyaluronan; lane 2, normal fascia (NF) GAGs precipitated with $27.5 \%$ ethanol; lane 3, NF GAGs precipitated with $50 \%$ ethanol; lane 5, Dupuytren's fascia (DF) GAGs precipitated with $27.5 \%$ ethanol; lane 6, DF GAGs precipitated with $50 \%$ ethanol. It is worth mentioning that in the case of DF samples non-precipitable material still contained significant amount of species showing electrophoretic mobility similar to that of reference DS.

ples and from 8.5 to $24 \mathrm{kDa}$ for DF specimens (Fig. $2 \mathrm{~b}$, lanes 1,2$)$.

The comparison of epimerization patterns of DS chains from NF and DF was carried out as follows. DS chain sections comprising mainly "iduronate disaccharides" as well as the clusters of "glucuronate disaccharides" were obtained after the action of testicular hyaluronidase and chondroitinase $B$, respectively. The former enzyme hydrolyzes ( $\beta 1$ 4) linkages between $\mathrm{N}$-acetyl-galactosamine and glucuronate (GlcA) residues localized in sequences of at least two GlcA repeat disaccharides (Hampson \& Gallagher, 1984). Thus the enzyme degrades CS and clusters of "glucuronate disaccharides" in DS chains. Moreover, hyaluronan (HA) is cleaved by hyaluronidase (Hampson \& Gallagher, 1984). In contrast, DS chain sections containing disaccharides with iduronate (IdoA) residue and/or single disaccharides with GlcA residue are resistant to the enzyme action.

Chondroitinase B splits $N$-acetyl-galactosamine-IdoA links while $N$-acetyl-galactosamineGlcA bonds are resistant to degradation (Gu et al., 1995). As CS comprises exclusively "glucuronate disaccharides" it is completely resistant to this enzyme. To eliminate the interference of CS present in fractions II derived from both fascia types, DS was extracted from these fraction samples by selec- tive precipitation (Fig. 3, lanes 2, 3, 5, 6) before enzyme treatment.

All obtained samples of DS degradation products were subjected to gradient-PAGE followed by Azure A/ammoniacal silver staining. Fig. 4a and c present typical ladder-like electrophoretic patterns of products obtained after hyaluronidase action, respectively, on fraction I DS and fraction II DS derived from both NF and DF. As can be seen, regardless of the tissue and fraction type, DSs revealed significant resistance to the enzyme as judged from the comparison of electrophoretic profiles of degradation products and intact chains (Fig. 4a and c versus Fig. 2). In contrast, simultaneously performed depolymerization of standard CS (Fig. 4b) and HA (not shown) by hyaluronidase yielded, respectively, a few species or a single one moving near the front of the resolution. Thus it may be concluded that contribution of regions containing mainly "iduronate disaccharides" to the composition of DS chains from both NF and DF is significant. These sections of fraction I DS chains displayed complex electrophoretic patterns with great numbers of subtle, closely located bands (Fig. 4a). In contrast, the enzyme resistant fragments of fraction II DSs formed patterns with prominent major bands between which only single species with lower mobility were interspersed (Fig. 4c). This electrophoretic behavior suggests that hyaluronidase resistant sections of fraction I DSs have more differentiated and lower sulfation degree than such regions from fraction II DSs.

Despite minor if any qualitative dissimilarities, the densitometric analysis of electrophoretic patterns revealed quantitative differences between products of NF and DF dermatan sulfate degradation by hyaluronidase. All DF samples displayed enhanced amounts of the enzyme resistant chain fragments showing intermediate and high electrophoretic mobility (Fig. 4d and Fig. 5). These results suggest alterations in DS epimerization pattern, also including an increase in CS-like structure contribution. To verify the latter assumption samples of fraction I and II were submitted to chondroitinase B action. Typical electrophoretic patterns of the enzyme resistant DS chain sections are presented in Fig. 6a and $\mathrm{c}$. The analysis of these profiles has revealed that contribution of chondroitinase $\mathrm{B}$ resistant regions to the composition of DSs from both NF and DF is significant (Fig. 7). Moreover, almost all chondroitinase B resistant chain regions included blocks of "glucuronate disaccharides" as judged by their sensitivity to chondroitinase AC I (Fig. $6 \mathrm{~b}$ and d). Thus DS chains from both NF and DF have a hybrid DS-CS structure within which most of "glucuronate sections" display rather small size as can be concluded from the fact that the majority of chondroitinase B resistant chain fragments showed high electrophoretic mobilities 

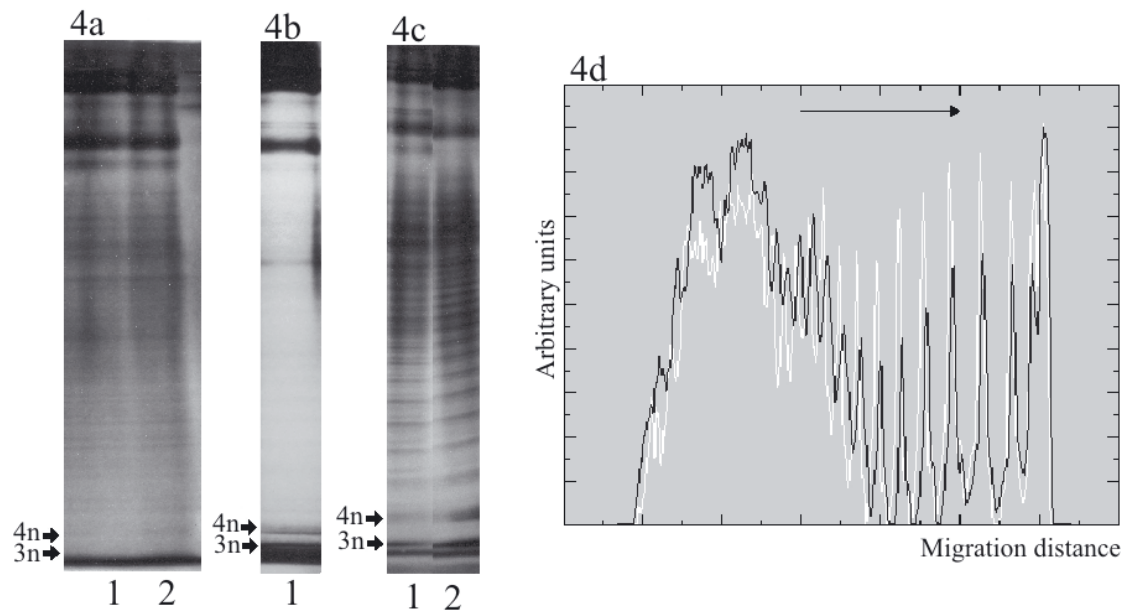

Figure 4a-d. Analysis of sensitivity of dermatan sulfate (DS) chains from normal fascia (NF) and Dupuytren's fascia (DF) to testicular hyaluronidase.

The products resistant to the enzyme were resolved by gradient-gel-electrophoresis followed by Azure A/ammoniacal silver staining. (4a) Fraction I DS chain fragments resistant to testicular hyaluronidase: lane 1, products of hydrolysis of NF sample $(0.75 \mu \mathrm{g}$ of hexuronic acids of DS); lane 2, products of hydrolysis of DF sample $(0.75 \mu \mathrm{g}$ of hexuronic acids of DS). (4b) Products of testicular hyaluronidase action on standard chondroitin-4-sulfate. (4c) Fraction II DS chain fragments resistant to testicular hyaluronidase: lane 1, products of hydrolysis of NF sample (1.0 $\mu \mathrm{g}$ of hexuronic acids of DS); lane 2, products of hydrolysis of DF sample $(1.0 \mu \mathrm{g}$ of hexuronic acids of DS). The arrows indicate migration positions of saccharides (most probably hexa- (3n) and octasaccharides (4n)) obtained by the complete degradation of standard chondroitin-4-sulfate by testicular hyaluronidase. (4d) Densitometric scans of products obtained after hyaluronidase action on fraction II DSs from NF (black line) and DF (white line). The arrow indicates the direction of electrophoretic migration.

(Fig. 6a and c). However, differences in electrophoretic patterns of such regions derived from fraction I and fraction II DSs were found (Fig. 6a and c) resembling those observed for hyaluronidase generated products (Fig. 4a and c) and suggesting differences in sulfation patterns.

Densitometric analysis of electrophoretic profiles of chondroitinase B resistant oligosaccharides indicates that irrespectively of fascia type, DS chains from fractions I and II differ in respect of the content of "glucuronate disaccharide" blocks (Fig. 7). Moreover, the obtained results confirmed our assumption that DS from DF contains more such chain sections than control GAG (Fig. 7). This phenomenon refers mainly to "glucuronate regions" of small and intermediate size (Fig. 6e).

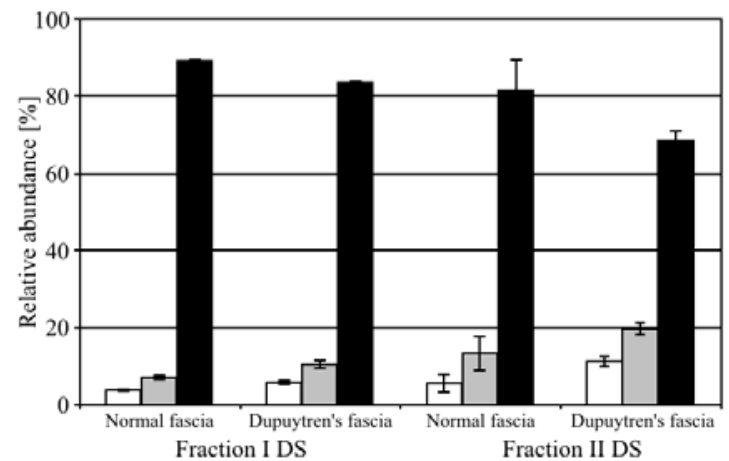

\section{DISCUSSION}

Our present study shows that NF derived DS chains display remarkable structural heterogeneity. However, the degree of structural complexity increases further in the case of DS from DF fibrous cords. The observed alterations of both qualitative and quantitative nature are reflected (i) in a distinct profile of the GAG chain molecular masses, (ii) different glucuronosyl epimerization pattern with lesser extent of the modification concomitant with the reduction of "glucuronate region" size and higher dispersion of such regions along the DS chain and (iii) in the increased frequency of oversulfated chains. The latter conclusion results from the higher proportion of frac-

Figure 5. Relative abundance of hyaluronidase resistant fragments in dermatan sulfate (DS) chains derived from normal (NF) and Dupuytren's fascia (DF).

The percentage content of particular chain fragments was determined by densitometric analysis of enzyme resistant product electrophoretic profiles. Error bars show the mean \pm S.D. of analysis of five and seven samples of NF and $\mathrm{DF}$, respectively. White bars, oligosaccharides migrating as two bands of high electrophoretic mobility corresponding to that of the products of complete degradation of standard chondroitin sulfate by hyaluronidase; grey bars, oligosaccharides of intermediate electrophoretic mobility (next four bands); black bars, remaining oligosaccharides. 

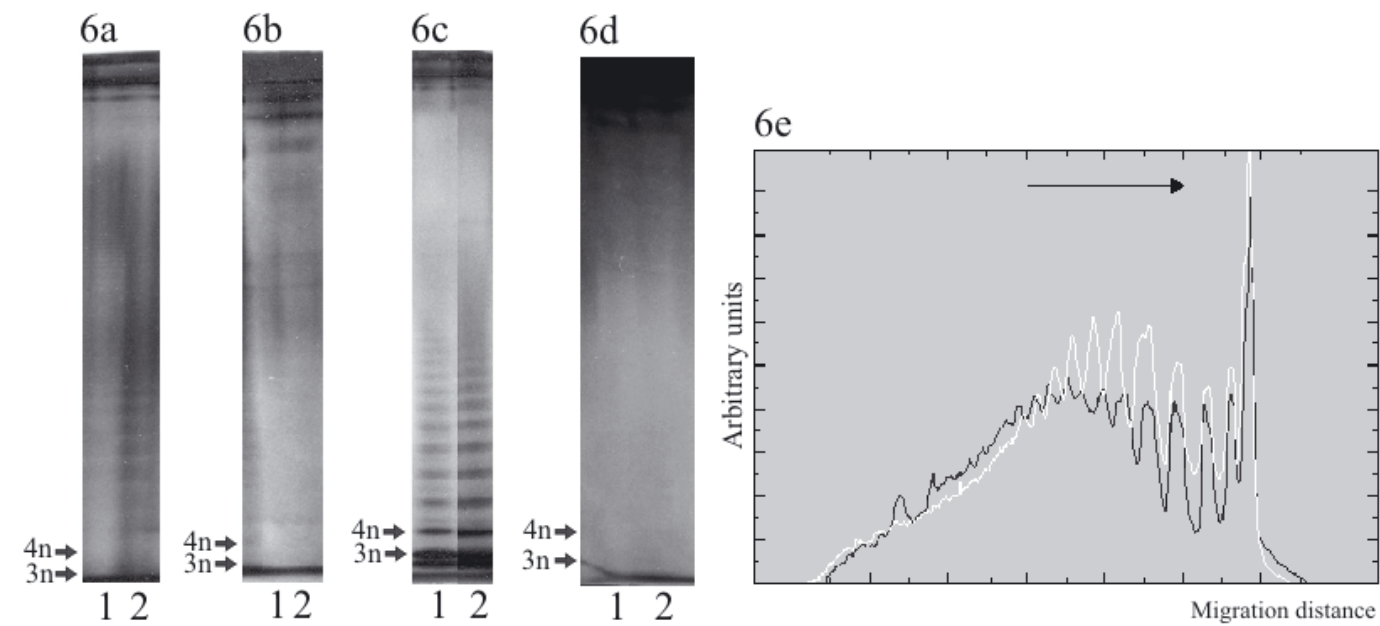

Figure 6a-e. Analysis of sensitivity of dermatan sulfate (DS) chains from normal fascia (NF) and Dupuytren's fascia (DF) to chondroitinase $B$.

The products resistant to the enzyme were resolved by gradient-gel-electrophoresis followed by Azure A/ammoniacal silver staining. (6a) Fraction I DS chain fragments resistant to chondroitinase B: lane 1, products of degradation of NF sample $(0.75 \mu \mathrm{g}$ of hexuronic acids of DS); lane 2, products of degradation of DF sample (0.75 $\mu \mathrm{g}$ of hexuronic acids of DS). (6b) Fraction I DS chain fragments resistant to combined action of chondroitinases B and AC I: lane 1, products of depolymerization of NF sample; lane 2, products of degradation of DF sample. (6c) Fraction II DS chain fragments resistant to chondroitinase B: lane 1, products of degradation of NF sample (1.25 $\mathrm{g}$ g of hexuronic acids of DS); lane 2, products of degradation of DF sample (1.25 $\mu \mathrm{g}$ of hexuronic acids of DS). (6d) Fraction II DS chain regions resistant to combined action of chondroitinases B and AC I: lane 1, products of depolymerization of NF sample; lane 2, products of degradation of DF sample. The arrows indicate migration positions of saccharides (most probably hexa- (3n) and octasaccharides $(4 n)$ ) obtained by the complete degradation of standard chondroitin-4-sulfate by testicular hyaluronidase. (6e) Densitometric scans of products obtained after chondroitinase B action on fraction II DS from NF (black line) and DF (white line). The arrow indicates the direction of electrophoretic migration.

tion II DS in relation to fraction I GAG found in DF as compared to normal tissue. Moreover, our suggestion concerning the oversulfation of DF derived DS is confirmed by the results of Tunn et al.

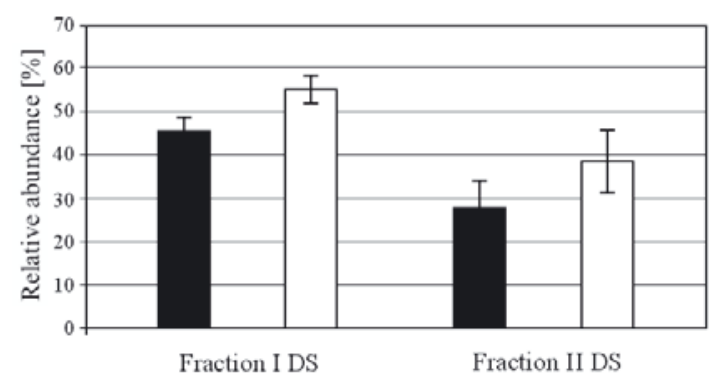

Figure 7. Relative abundance of "glucuronate disaccharide" blocks in dermatan sulfate (DS) chains derived from normal (NF) and Dupuytren's fascia (DF).

The percentage content of these regions was determined as the ratio of the area under densitometric scan of chondroitinase $B$ resistant chain fragments and the total area under densitometric scans of chondroitinase B and hyaluronidase resistant chain sections. Error bars show the mean \pm S.D. of analysis of five and seven samples of NF and DF, respectively. Black bars, contribution of "glucuronate regions" to DS chains from NF; white bars, contribution of "glucuronate regions" to DS chains from DF. Differences in "glucuronate section" relative contents except those between fraction II DS from DF and fraction I DS from NF were statistically significant $(P<0.05)$.
(1988), who found increased content of sulfated disaccharides generated by chondroitinase $A B C$ action on the GAG from such tissue samples. The profound remodeling of DS profile also involving an increase in this GAG content, observed by us in advanced DC, may be due to two reasons: (i) alterations in expression of particular DSPGs manifested most probably by enhanced biosynthesis of small leucine rich PG biglycan (Koźma et al., 2005) as well as (ii) changes in activity of GAG modifying enzymes. In the course of DC, the DSPG biosynthesis is most probably attributed to tissue myofibroblasts (Tomasek et al., 1999) and is under control of growth factors. PDGF and TGF $\beta$ implicated in DC pathogenesis (Badalamente \& Hurst, 1999) are known to influence DSPG expression and to induce the augmentation of GAG chain size (Kähäri et al., 1991; Schönherr et al., 1997). Moreover, the study of Tiedemann et al. (2005) revealed that TGF $\beta-1$ alone and especially in combination with PDGF BB (and epidermal growth factor) reduced the activity of glucuronosyl 5 '-epimerase and stimulated some sulfatase expression in cultured human lung fibroblasts. On the other hand, DS remodeling associated with advanced DC resembles these alterations found in tubulointerstitial nephritis and liver cirrhosis (Koshiishi et al., 
1999; 2002) thus being the hallmark of myofibroblast activity irrespective of fibrosis process localization.

As it results from our present study referring to the total fascia DS and from our previous examination concerning fascia DSPGs extractable with urea (Koźma et al., 2005), in both NF and DF two DSPG pools may exist. The minor one, comprising about $27 \%$ and $34 \%$ of total DS content in the case of NF and DF, respectively, includes molecules rather weakly bound to other ECM components and extractable with chaotropic agents. In contrast, the second pool DSPGs are tightly anchored to the matrix network most probably by cross-links formed with participation of enzymes such as transglutaminase (Kinsella \& Wight, 1990). However, these both pool DSPGs seem to bear GAG chains with a similar extent of glucuronosyl epimerization. This conclusion can be drawn from the fact that the average content of "glucuronate regions" calculated for urea extractable DS (Koźma et al., 2005) and that found in the present study for total DS are similar, achieving levels of about $32 \%$ and $40 \%$ in the case of NF and $\mathrm{DF}$, respectively. Thus, "glucuronate sections" contribution to DS chain composition does not seem to be responsible for the GAG distribution between two fascia pools of DSPGs both under physiological and fibrosis conditions. Nevertheless, it can not be excluded that DS chains with similar epimerization extents may differ in respect to "iduronate" and "glucuronate section" arrangement that in turn can modify ligand affinity of parent DSPG and, as a consequence, their different matrix localization and susceptibility to cross-link formation. Interestingly, we have found that total DS has distinct content of smaller size "glucuronate sections" than the GAG extractable with urea (Koźma et al., 2005) both in the case of NF and DF samples.

The remodeling of Dupuytren's fascia DS profile observed by us may have severe consequences for matrix properties and cell behavior though the detailed significance is still poorly known. DS chains are most frequently the components of small leucine rich matrix PGs decorin and biglycan which participate in matrix assembly by influencing collagen fibrillogenesis (Corsi et al., 2002). In this process, DS chains of PG molecules non-covalently bound to the surface of collagen fibrils are responsible for fibril packing by forming anti-parallel duplexes which bridge these collagen structures (Scott, 2003). Then, DC associated DSPG remodeling, in particular an increase in frequency of DS chains with small size, may promote the formation of a tight tendon like collagen network characteristic for the disease (Rayan, 1999). Moreover, decorin most probably by its DS chain can also stimulate fibronectin fibrillogenesis (Kinsella et al., 2000; Gendelman et al., 2003) that controls both collagen deposition and integrin dependent intracellular signaling involved in the regulation of cell motility, proliferation and survival (Sottile \& Hocking, 2002). Interestingly, increased fibronectin deposition is a key feature of DC pathogenesis (Tomasek et al., 1986). This disease is also characterized by elastogenesis impairment (Gaździk et al., 1984) that may be due to excessive DS accumulation in the pericellular compartment. As is shown from the study of Hinek and Wilson (2000), pericellular DS is responsible for disturbances in metabolism of an elastin-binding protein that plays an essential role in proper cellular trafficking and the secretion of tropoelastin molecules. Finally, DS interacts with many growth factors, including fibroblast growth factor-2 (FGF-2) and BB isoform of PDGF also implicated in DC (Badalamente \& Hurst, 1999). DS binding of FGF-2 can be involved in ECM sequestration of the growth factor as well as in promoting its proliferative activity (Nandini et al., 2004; 2005; Taylor et al., 2005). The former function seems to be attributed to DS chains with rather short "iduronate sections" scattered between "glucuronate sections" also of small size (Nandini et al., 2004; 2005). In contrast, DS chains with long "iduronate disaccharide" blocks seem to be preferentially implicated in the stimulation of proliferative activity of FGF-2 (Taylor et al., 2005). In addition to FGF-2 binding, DS interaction with long isoform of PDGF BB also needs "iduronate regions" (Garcia-Olivas et $a l ., 2003)$. In contrast, binding of PDGF BB short isoform which is a product of long molecule proteolytic processing, seems to prefer blocks of "glucuronate disaccharides" (Garcia-Olivas et al., 2003). Moreover, it should be emphasized that DS, particularly existing in larger amounts, can effectively compete cell membrane localized PDGF acceptors in growth factor binding (Garcia-Olivas et al., 2003) Then, Dupuytren's disease associated with remodeling of DS chain structure can influence both the bioavailability and activity of growth factors thus modifying fascia cell functions.

Hence, it can be concluded that alterations in fascia DS profile though they represent only a part of complex tissue remodeling associated with DC may be a significant element of disease pathogenesis.

\section{REFERENCES}

Badalamente MA, Hurst LC (1999) The biochemistry of Dupuytren's disease. Hand Clin 15: 35-42.

Blumenkrantz N, Asboe-Hansen G (1973) New method for determination of uronic acids. Anal Biochem 54: 484489.

Casu B, Ferro DR, Ragazzi M, Torri G (1993) Conformation of iduronic acid-containing glycosaminoglycans. In Dermatan sulphate proteoglycans chemistry, biology, chemi- 
cal pathology. Scott JE, ed, pp 41-53. Portland Press, London, Chapel Hill.

Corsi A, Xu T, Chen X-D, Boyde A, Liang J, Mankani M, Sommer B, Iozzo VR, Eichstetter I, Robey PG, Bianco P, Young MF (2002) Phenotypic effects of biglycan deficiency are linked to collagen fibril abnormalities, are synergized by decorin deficiency, and mimic EhlersDanlos-like changes in bone and other connective tissues. J Bone Miner Res 17: 1180-1189.

Cowman MK, Slahetka MF, Hittner DM, Kim J, Forino M, Gadelrab G (1984) Polyacrylamide-gel electrophoresis and Alcian blue staining of sulphated glycosaminoglycan oligosaccharides. Biochem J 221: 707-716.

Dal Cin P, De Smet L, Sciot R, Van Damme B, Van den Berghe H (1999) Trisomy 7 and trisomy 8 in dividing and non-dividing tumor cells in Dupuytren's disease. Cancer Genet Cytogenet 108: 137-140.

Flint MH, Gillard GC, Reilly HC (1982) The glycosaminoglycans of Dupuytren's disease. Connect Tissue Res 9: 173-179.

Garcia-Olivas R, Hoebeke J, Castel S, Reina M, Fager G, Lustig F, Vilaro S (2003) Differential binding of platelet-derived growth factor isoforms to glycosaminoglycans. Histochem Cell Biol 120: 371-382.

Gaździk T, Januszewski K, Jędrysik M (1984) Micromorphological studies in Dupuytren's contracture. Chir Narz Ruchu Ortop Pol 49: 345-349 (in Polish).

Gendelman R, Burton-Wurster NI, MacLeod JN, Lust G (2003) The cartilage-specific fibronectin isoform has a high affinity binding site for the small proteoglycan decorin. J Biol Chem 278: 11175-11181.

Głowacki A, Olczyk K, Sonecki P, Koźma E, Najmiec T (1994) Glycosaminoglycans of normal and scarred fascia. Acta Biochim Polon 41: 166-169.

Gu K, Linhardt RJ, Laliberte M, Gu K, Zimmermann DR (1995) Purification, characterization and specificity of chondroitin lyases and glucuronidases from Flavobacterium heparinum. Biochem J 312: 569-577.

Gudmundsson KG, Arngrimsson R, Sigfusson N, Bjornsson A, Jonsson T (2000) Epidemiology of Dupuytren's disease: clinical, serological, and social assessment. J Clin Epidemiol 53: 291-296.

Hamai A, Hashimoto N, Mochizuki H, Kato F, Makiguchi Y, Horie K, Suzuki S (1997) Two distinct chondroitin sulfate $\mathrm{ABC}$ lyases. An endoeliminase yielding tetrasaccharides and an exoeliminase preferentially acting on oligosaccharides. J Biol Chem 272: 9123-9130.

Hampson IN, Gallagher JT (1984) Separation of radiolabelled glycosaminoglycan oligosaccharides by polyacrylamide-gel electrophoresis. Biochem J 221: 697-705.

Hinek A, Wilson SE (2000) Impaired elastogenesis in Hurler disease. Dermatan sulfate accumulation linked to deficiency in elastin-binding protein and elastic fiber assembly. Am J Pathol 156: 925-938.

Jung Y, Hohmann TC, Gerneth JA, Novak J, Wasserman RC, D'Andrea BJ, Newton RH, Danowski TS (1971) Diabetic hand syndrome. Metabolism 20: 1008-1015.

Kähäri VM, Larjava H, Uitto J (1991) Differential regulation of extracellular matrix proteoglycan (PG) gene expression. Transforming growth factor-beta 1 up-regulates biglycan (PGI), and versican (large fibroblast PG) but down-regulates decorin (PGII) mRNA levels in human fibroblasts in culture. J Biol Chem 266: 10608-10615.

Kinsella MG, Bressler SL, Wight TN (2004) The regulated synthesis of versican, decorin, and biglycan: extracellular matrix proteoglycans that influence cellular phenotype. Crit Rev Eukaryot Gene Expr 14: 203-234.

Kinsella MG, Fischer JW, Mason DP, Wight TN (2000) Retrovirally mediated expression of decorin by macrov- ascular endothelial cells. Effects on cellular migration and fibronectin fibrillogenesis in vitro. J Biol Chem 275: 13924-13932.

Kinsella MG, Wight TN (1990) Formation of high molecular weight dermatan sulfate proteoglycan in bovine aortic endothelial cell cultures. Evidence for transglutaminase-catalyzed cross-linking to fibronectin. J Biol Chem 265: 17891-17898.

Koshiishi I, Hasegawa T, Imanari T (2002) Quantitative and qualitative alterations of chondroitin/dermatan sulfates accompanied with development of tubulointerstitial nephritis. Arch Biochem Biophys 401: 38-43.

Koshiishi I, Takenouchi M, Imanari T (1999) Structural characteristics of oversulfated chondroitin/dermatan sulfates in the fibrous lesions of the liver with cirrhosis. Arch Biochem Biophys 370: 151-155.

Koźma EM, Olczyk K, Głowacki A (2001) Dermatan sulfates of normal and scarred fascia. Comp Biochem Physiol Part B 128: 221-232.

Koźma EM, Olczyk K, Głowacki A, Bobiński R (2000) An accumulation of proteoglycans in scarred fascia. Mol Cell Biochem 203: 103-112.

Koźma EM, Olczyk K, Wisowski G, Głowacki A, Bobiński $R$ (2005) Alterations in the extracellular matrix proteoglycan profile in Dupuytren's contracture affect the palmar fascia. J Biochem 137: 468-476.

Lennox IAC, Murali SR, Porter R (1993) A study of the repeatability of the diagnosis of Dupuytren's contracture and its prevalence in the Grampian Region. J Hand Surg 18B: 258-261.

Lyon M, Gallagher JT (1990) A general method for the detection and mapping of submicrogram quantities of glycosaminoglycan oligosaccharides on polyacrylamide gels by sequential staining with azur $\mathrm{A}$ and ammoniacal silver. Anal Biochem 185: 63-70.

Nandini CD, Itoh N, Sugahara K (2005) Novel 70-kDa chondroitin sulfate/dermatan sulfate hybrid chains with a unique heterogenous sulfation pattern from shark skin, which exhibit neuritogenic activity and binding activities for growth factors and neurotrophic factors. J Biol Chem 280: 4058-4069.

Nandini CD, Mikami T, Ohta M, Itoh N, Akiyama-Nambu F, Sugahara K (2004) Structural and functional characterization of oversulfated chondroitin sulfate/dermatan sulfate hybrid chains from the notochord of hagfish. $J$ Biol Chem 279: 50799-50809.

Prydz K, Dalen KT (2000) Synthesis and sorting of proteoglycans. J Cell Sci 113: 193-205.

Rayan GM (1999) Clinical presentation and types of Dupuytren's disease. Hand Clin 15: 87-96.

Roden L, Baker JR, Cifonelli JA, Mathews MB (1972) Isolation and characterization of connective tissue polysaccharides. Methods Enzymol 28: 73-140.

Rombouts JJ, Noel H, Legrain Y, Munting E (1989) Prediction of recurrence in the treatment of Dupuytren's disease: Evaluation of a histologic classification. J Hand Surg 14: 644-652.

Schönherr E, Kinsella MG, Wight TN (1997) Genistein selectively inhibits platelet-derived growth factor stimulated versican biosynthesis in monkey arterial smooth muscle cells. Arch Biochem Biophys 339: 353-361.

Scott JE (2003) Elasticity in extracellular matrix „shape modules" of tendon, cartilage, etc. A sliding proteoglycan-filament model. J Physiol 553: 335-343.

Sottile J, Hocking DC (2002) Fibronectin polymerization regulates the composition and stability of extracellular matrix fibrils and cell-matrix adhesion. Mol Biol Cell 13: 3546-3559. 
Taylor KR, Rudisill JA, Gallo RL (2005) Structural and sequence motifs in dermatan sulfate for promoting fibroblast growth factor-2 (FGF-2) and FGF-7 activity. J Biol Chem 280: 5300-5306.

Tiedemann K, Olander B, Eklund E, Todorova L, Bengtsson M, Maccarana M, Westergren-Thorsson G, Malmstrom A (2005) Regulation of the chondroitin/dermatan fine structure by transforming growth factor- $\beta 1$ through effects on polymer-modifying enzymes. Glycobiology 15: 1277-1285.

Tomasek JJ, Schultz RJ, Episalla CW, Newman SA (1986) The cytoskeleton and extracellular matrix of the Dupuytren's disease "myofibroblast": An immunofluores- cence study of a nonmuscle cell type. J Hand Surg Am 11: $365-371$.

Tomasek JJ, Vaughan MB, Haaksma CJ (1999) Cellular structure and biology of Dupuytren's disease. Hand Clin 15: 21-34.

Tunn S, Gurr E, Delbruck A, Buhr T, Flory J (1988) The distribution of unsulphated and sulphated glycosaminoglycans in palmar fascia from patients with Dupuytren's disease and healthy subjects. J Clin Chem Clin Biochem 26: 7-14.

Wegrowski Y, Maquart FX (2004) Involvement of stromal proteoglycans in tumor progression. Crit Rev Oncol Hematol 49: 259-268. 